\title{
Definitional Differences of 'Outpatient' Versus 'Inpatient' THA and TKA Can Affect Study Outcomes
}

\author{
Patawut Bovonratwet BS, Matthew L. Webb MD, MHS, Nathaniel T. Ondeck BS, \\ Adam M. Lukasiewicz MD, MSc, Jonathan J. Cui BS, Ryan P. McLynn BS, \\ Jonathan N. Grauer MD
}

Published online: 12 January 2017

(C) The Association of Bone and Joint Surgeons (B) 2017

\begin{abstract}
Background There has been great interest in performing outpatient THA and TKA. Studies have compared such procedures done as outpatients versus inpatients. However, stated "outpatient" status as defined by large national databases such as the National Surgical Quality Improvement Program (NSQIP) may not be a consistent entity, and the actual lengths of stay of those patients categorized as outpatients in NSQIP have not been specifically ascertained and may in fact include some patients who are "observed" for one or more nights. Current regulations in the United States allow these "observed" patients to stay more than one night at the hospital under observation status despite being coded as outpatients. Determining the degree
\end{abstract}

One of the authors certifies that he (PB), or a member of his immediate family, has or may receive payments or benefits, during the study period an amount less than USD 10,000 from Yale School of Medicine Medical Student Fellowship (New Haven, CT, USA), all outside the submitted work. One of the authors certifies that he (JNG), or a member of his immediate family, has or may receive payments or benefits, during the study period an amount less than USD 10,000 from Bioventus (Durham, NC, USA), an amount less than USD 10,000 from ISTO Technologies (St Louis, MO, USA), an amount less than USD 10,000 from Medtronic (Minneapolis, MN, USA), an amount less than USD 10,000 from Stryker (Kalamazoo, MI, USA), an amount less than USD 10,000 from Novella Clinical (Morrisville, NC, USA), an amount less than USD 10,000 from Andante Medical Devices (White Plains, NY, USA), an amount less than USD 10,000 from Vertex Pharmaceuticals (Boston, MA, USA), and an amount less than USD 10,000 from the Orthopaedic Trauma Association (Rosemont, IL, USA), all outside the submitted work.

All ICMJE Conflict of Interest Forms for authors and Clinical Orthopaedics and Related Research ${ }^{\mathbb{R}}$ editors and board members are on file with the publication and can be viewed on request.

Clinical Orthopaedics and Related Research ${ }^{\mathbb{R}}$ neither advocates nor endorses the use of any treatment, drug, or device. Readers are encouraged to always seek additional information, including FDAapproval status, of any drug or device prior to clinical use. to which this is the case, and what, exactly, "outpatient" means in the NSQIP, may influence the way clinicians read studies from that source and the way hospital systems and policymakers use those data.

Questions/Purposes The purposes of this study were (1) to utilize the NSQIP database to characterize the differences in definition of "inpatient" and "outpatient" (stated status versus actual length of stay [LOS], measured in days) for THA and TKA; and (2) to study the effect of defining populations using different definitions.

Methods Patients who underwent THA and TKA in the 2005 to 2014 NSQIP database were identified. Outpatient procedures were defined as either hospital LOS $=0$ days in NSQIP or being termed "outpatient" by the hospital.

Each author certifies that his institution approved or waived approval for the human protocol for this investigation and that all investigations were conducted in conformity with ethical principles of research.

This work was performed at Yale School of Medicine, New Haven, CT, USA.

Electronic supplementary material The online version of this article (doi:10.1007/s11999-017-5236-6) contains supplementary material, which is available to authorized users.

P. Bovonratwet, N. T. Ondeck, A. M. Lukasiewicz, J. J. Cui, R. P. McLynn, J. N. Grauer ( $\square)$

Department of Orthopaedics and Rehabilitation, Yale University School of Medicine, 47 College Street, New Haven, CT 06520 , USA

e-mail: jonathan.grauer@yale.edu

M. L. Webb

Department of Orthopaedic Surgery, Hospital of the University of Pennsylvania, Philadelphia, PA, USA 
The actual hospital LOS of "outpatients" was characterized. "Outpatients" were considered to have stayed overnight if they had a LOS of 1 day or longer. The effects of the different definitions on 30-day outcomes were evaluated using multivariate analysis while controlling for potential confounding factors.

Results Of 72,651 patients undergoing THA, 529 were identified as "outpatients" but only 63 of these $(12 \%)$ had a LOS $=0$. Of 117,454 patients undergoing TKA, 890 were identified as "outpatients" but only 95 of these $(11 \%)$ had a LOS $=0$. After controlling for potential confounding factors such as gender, body mass index, functional status before surgery, comorbidities, and smoking status, we found "inpatient" THA to be associated with increased risk of any adverse event (relative risk, 2.643, $\mathrm{p}=0.002$ ), serious adverse event (relative risk, 2.455, $\mathrm{p}=0.011$ ), and readmission (relative risk, 2.775, $\mathrm{p}=0.010$ ) compared with "outpatient" THA. However, for the same procedure and controlling for the same factors, patients who had LOS $>0$ were not associated with any increased risk compared with patients who had $\operatorname{LOS}=0$. A similar trend was also found in the TKA cohort.

Conclusions Future THA, TKA, or other investigations on this topic should consistently quantify the term "outpatient" because different definitions, stated status or actual LOS, may lead to different assignments of risk factors for postoperative complications. Accurate data regarding risk factors for complications after total joint arthroplasty are crucial for efforts to reduce length of hospital stay and minimize complications.

Level of Evidence Level III, therapeutic study.

\section{Introduction}

The annual demand for both THA and TKA in the United States is expected to increase substantially over the next decade owing to an aging population and increased public demand [26, 29]. There has been a recent surge of interest in performing THA and TKA in the outpatient setting $[3,4,9]$. The benefits of outpatient arthroplasty are reported to include higher patient satisfaction and reduced costs [15, 29]. Multiple studies comparing the outcomes of inpatient with outpatient arthroplasty have been reported $[2,5,9,10,15,16,24,25,29,30,37,40,48]$.

However, the difference between "inpatient" and "outpatient" is not as intuitive as one might think. Outpatient surgery is generally thought of as surgery for which patients are discharged home on the same day as the procedure. That said, current regulations in the United States allow patients who underwent outpatient surgery to stay a night or more at the hospital under "observation" status despite being coded as outpatients. This "observation" status obscures the division between what is considered inpatient or outpatient. Some studies on outpatient arthroplasty have defined "outpatient" status as patients who were discharged the same day $[5,9,10]$, patients who stayed at most one night at the hospital [2, 4, 24], patients who stayed at most two nights at the hospital [15, 25], or simply provided stated status with no quantitative description $[16,29,40]$.

Since 2005, the number of orthopaedic studies reported that utilize national databases has risen considerably [7]. These databases allow access to large, nationally representative populations and grant high statistical power to study less common procedures and rare outcomes. One commonly used national database in orthopaedics is the American College of Surgeons National Surgical Quality Improvement Program (NSQIP). NSQIP gathers highquality data from over 500 participating institutions in the United States and tracks general health complications for 30 days postoperatively [1]. This data set has proved to be a valid data source in orthopaedic research [47]. The NSQIP database allows investigators to easily compare inpatient and outpatient procedures because it provides an "inpatient" or "outpatient" status variable. Many studies in different surgical subspecialties have used this variable to compare short-term outcomes of inpatient versus outpatient surgery [19-22, 27, 28, 33, 34, 36, 39, 42-46]. Multiple recent studies in orthopaedics have used these variables to study outpatient procedures or have included these variables in their studies as well [32, 35, 41, 50]. However, in NSQIP, the "inpatient" or "outpatient" variable is distinct from the hospital length of stay (LOS) variable [1]. On analyzing these two distinct variables, we found that the "inpatient" or "outpatient" variable often does not match with LOS $>0$ day or LOS $=0$ day. Determining the degree to which this is the case, and what, exactly, "outpatient" means in NSQIP, may influence the way clinicians interpret results from that source and the way hospital systems and policymakers use those data.

The primary purpose of this investigation is to characterize the different definitions of inpatient and outpatient status for THAs and TKAs by utilizing the NSQIP database. The secondary purpose is to determine the effects of using different definitions of inpatient and outpatient status on complications and readmissions to the hospital after surgery.

\section{Patients and Methods}

The NSQIP database collects over 300 patient variables through chart abstraction and patient interviewers from over 500 participating institutions in the United States [1]. The database uses trained clinical reviewers to collect 
specifically defined variables such as preoperative comorbidities, intraoperative variables, and postoperative adverse events for 30 days regardless of hospital discharge [23]. Interrater reliability audits revealed an overall disagreement rate of only approximately $2 \%$ for all assessed program variables [1]. This data set has been proven to be a valid data source in orthopaedic surgery [47]. Our institutional review board granted an exemption for studies using this data set.

Patients who underwent THAs and TKAs in 2005 to 2014 were extracted from NSQIP by using Current Procedural Classification (CPT) codes. Patients undergoing THA were selected using CPT code 27130, whereas patients undergoing TKA were selected using CPT code 27447. Cases involving fractures, trauma, neoplasia, and infections were excluded by using International Classification of Diseases, 9th Revision codes. Emergency cases were excluded as well. Based on these criteria, 72,651 patients undergoing THA and 117,454 patients undergoing TKA remained for analysis.

Patient attributes such as age, gender, height, weight, functional status before surgery, and smoking status are available in the database. For each patient, a modified version of the Charlson Comorbidity Index (CCI) [8] that has been adapted to the NSQIP database was calculated [12]. Modified CCIs have been shown to predict similar prognoses as the original CCI and have also been previously used with NSQIP data $[6,31,49]$. The comorbidities used to calculate the modified CCI include (followed by their CCI point values): congestive heart failure (1), myocardial infarction within the 6 months before surgery (1), peripheral vascular disease or rest pain (1), history of transient ischemic attack or cerebrovascular accident (1), diabetes mellitus (1), chronic obstructive pulmonary disease (1), end-stage renal disease (2), hemiplegia (2), ascites or esophageal varices (3), and disseminated cancer (6). The modified CCI for each case is then calculated by summing up these point values and adding a point for age of at least 40 years with an additional point for each decade older than 40 years old.

NSQIP provides a distinct "inpatient" or "outpatient" variable. According to the NSQIP Data User Guide, the "inpatient" and "outpatient" classification variable is defined as "The hospital's definition of inpatient and outpatient status" [1]. This means that each hospital that supplies clinical data to NSQIP will also give their patients' admission status based on their own definition of what is considered inpatient or outpatient, which could be different.

NSQIP also provides a LOS variable, recorded as days, and is defined as the length of hospital stay from hospital admission to discharge. A patient who had LOS $=0$ was considered to be discharged home the same day of surgery and did not stay overnight at the hospital. A patient who had LOS $>0$ was considered to have stayed at least one night at the hospital. Patients who are under 23-hour holds are part of the LOS $>0$ cohort. The maximum LOS in this study is limited to 30 days.

Individual adverse events are tracked during the first 30 postoperative days regardless of discharge by NSQIP [1]. These events were used to generate three classes of adverse events. A minor adverse event (MAE) was defined as the occurrence of any of the following: wound dehiscence, urinary tract infection, pneumonia, and renal insufficiency. A serious adverse event (SAE) was defined as the occurrence of any of the following: death, sepsis/septic shock, unplanned intubation, on ventilator $>48$ hours, stroke/ cerebrovascular event, cardiac arrest requiring cardiopulmonary resuscitation, myocardial infarction, acute renal failure, thromboembolic event, wound-related infection, and return to the operating room. Any adverse event was defined as the occurrence of a MAE or a SAE.

Readmission within 30 days for each patient is also recorded in NSQIP database. Occurrence of readmission is reported in NSQIP database for cases that occurred in 2011 to 2014 , but not for earlier cases.

Hospital LOS characteristics for both patients undergoing THA and those undergoing TKA were depicted by graphic representations. Patients who had "outpatient" or "inpatient" surgery according to the classification variable provided by NSQIP were analyzed for LOS $=0$ or LOS $>0$.

Both patients undergoing THA and those undergoing TKA were also subdivided into two subgroups, patients who had a LOS of 0 day and patients who had a LOS greater than 0 day. A Kaplan-Meier curve was used to plot total length of hospital stay for both the "outpatient" and "inpatient" groups for both types of surgery. The horizontal axis of these curves was plotted up to a 5-day LOS, because this captured $90 \%$ of the study population.

\section{Statistical Analyses}

For the multivariate analyses in this study, propensity score matching was used to match "inpatient" to "outpatient" and "LOS $>0$ " to "LOS $=0$ " candidates. Propensity score matching was used to minimize the effects of confounding related to differences in patient demographics as a result of the nonrandom assignment of patients to outpatient or inpatient surgery. This type of matching uses a propensity score, which is a single score that is calculated based on covariate data, to match patients from different treatment groups [18]. In the current study, each "outpatient" and "LOS $=0$ " case was matched with one "inpatient" and "LOS $>0$ " case, respectively, without replacement with regard to gender, body mass index, modified CCI, functional status before surgery, and smoking status. Age was not adjusted for separately because age is already a part of 
modified CCI. Matching without replacement has been used to create matched cohorts for comparison of outpatient and inpatient orthopaedic procedures before. This type of matching tends to reduce bias toward poorer outcomes for the inpatient cohort [32, 41].

Two distinct sets of multivariate analyses, utilizing Poisson regressions with robust error variance, were then performed to calculate the relative risks of three adverse event classes and readmission for patients who underwent THA who had "inpatient" surgery relative to "outpatient" surgery and who had LOS $>0$ relative to LOS $=0$ [52]. In these multivariate analyses, 529 matched "inpatient" cases were compared with 529 "outpatient" cases and 436 matched LOS $>0$ cases were compared with 436 LOS $=0$ cases.

Another two distinct sets of multivariate analyses were performed to calculate the relative risks of three adverse event classes and readmission for patients who underwent TKA who had "inpatient" surgery relative to "outpatient" surgery and who had LOS $>0$ relative to LOS $=0$. The same type of regression was used to calculate the relative risks as the THA cohort. In these multivariate analyses, 890 matched "inpatient" cases were compared with 890 "outpatient" cases and 663 matched LOS $>0$ cases were compared with 663 LOS $=0$ cases.

Statistical analyses were conducted using Stata Version 13.0 (StataCorp, LP, College Station, TX, USA). For the multivariate analyses, a Bonferroni correction was used, and with $\mathrm{n}=4$, the level of significance was set at $\alpha=$ $0.0125[11]$.

\section{Results}

Characterizing the Definition of Inpatient and Outpatient Status for THA

We found that a large majority of patients (466 of 529 [88\%]) coded in NSQIP as "outpatient" THAs in fact stayed in the hospital for one or more nights. Of the patients undergoing "outpatient" THA, 63 had a LOS $=0$ day (12\%), whereas 466 had a LOS $>0$ day (88\%). Of the patients undergoing "inpatient" THA, 373 had a LOS $=0$ day $(0.52 \%)$, whereas 71,749 had a $\operatorname{LOS}>0(99 \%)$ (Fig. 1). Based on the exclusion criteria outlined, 72,651 patients undergoing THA were identified. Of these, 529 were identified as "outpatient" procedures $(0.73 \%)$ and 72,122 were identified as "inpatient" procedures (99\%). Demographics and comorbidities of patients who underwent THA are reported (Appendix 1 [Supplemental materials are available with the online version of $\operatorname{CORR}$ (.]).

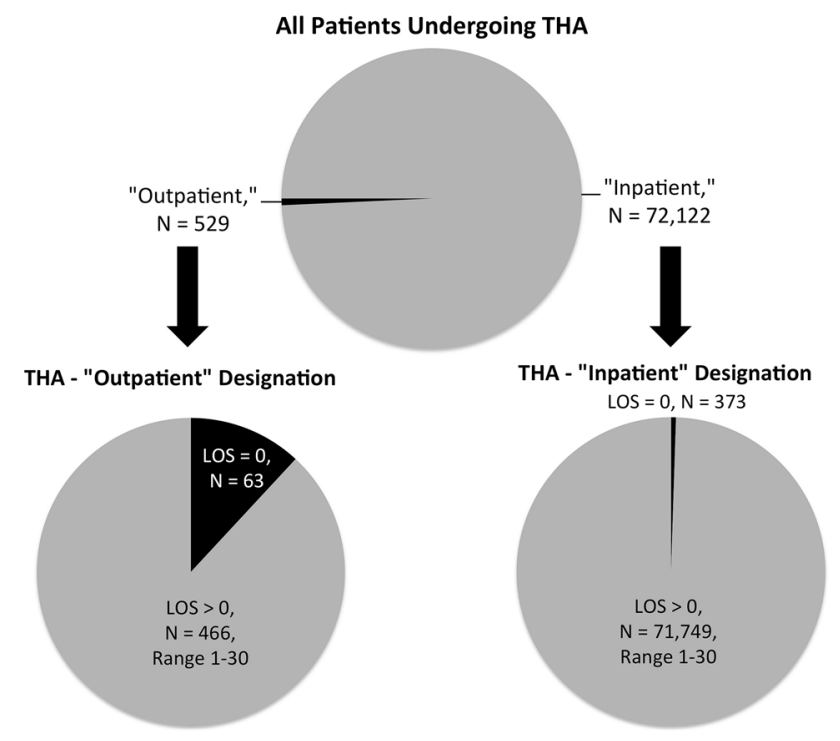

Fig. 1 Top pie chart shows the distribution of "inpatient" and "outpatient" THAs. Lower left pie chart shows the distribution of LOS of patients who received "outpatient" THA. Lower right pie chart shows the distribution of LOS of patients who received "inpatient" THA.

The Kaplan-Meier curve revealed that $99 \%$ of all "inpatient" and $88 \%$ of all "outpatient" THA patients stayed at least one night and remained at the hospital at LOS $=1$ day. Furthermore, a total of $19 \%$ of all "inpatient" and $22 \%$ of all "outpatient" THA patients remained at the hospital at $\operatorname{LOS}=4$ days (Fig. 2).

Characterizing the Definition of Inpatient and Outpatient Status for TKA

Likewise, a large majority (795 of 890 [89\%]) of patients undergoing TKA described in the NSQIP database as having undergone "outpatient" surgery stayed in the hospital for at least one night. Of the patients undergoing "outpatient" TKA, 95 had a LOS $=0$ day $(11 \%)$, whereas 795 had a LOS $>0$ day (89\%). Of the patients undergoing "inpatient" TKA, 568 had a LOS $=0$ day $(0.49 \%)$, whereas 115,996 had a LOS $>0$ day (99.5\%) (Fig. 3). Based on the exclusion criteria outlined, 117,454 patients undergoing TKA were identified. Of these, 890 were identified as "outpatient" procedures $(0.76 \%)$ and 116,564 were identified as "inpatient" procedures (99\%). Demographics and comorbidities of patients who underwent TKA are reported (Appendix 1).

The Kaplan-Meier curve revealed that $99.5 \%$ of all "inpatient" and $89 \%$ of all "outpatient" TKA patients stayed at least one night and remained at the hospital at LOS $=1$ day. Furthermore, a total of $21 \%$ of all "inpatient" 


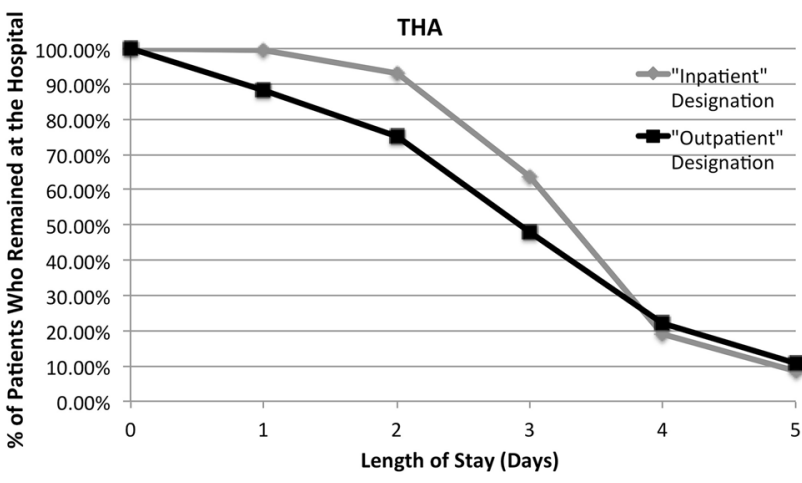

Fig. 2 Kaplan-Meier curve shows the percentage of patients who remained at the hospital at a particular LOS after receiving "inpatient" or "outpatient" THA. Y-axis represents the percentage of patients who remained at the hospital. X-axis represents the LOS.

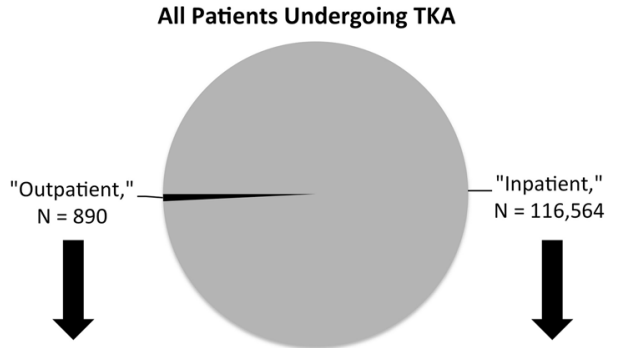

TKA - "Outpatient" Designation

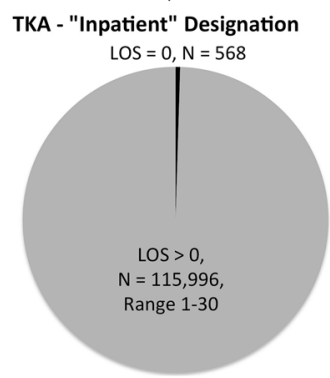

Fig. 3 Top pie chart shows the distribution of "inpatient" and "outpatient" TKAs. Lower left pie chart shows the distribution of LOS of patients who received "outpatient" TKA. Lower right pie chart shows the distribution of LOS of patients who received "inpatient" TKA.

and $23 \%$ of all "outpatient" TKA patients remained at the hospital at $\operatorname{LOS}=4$ days (Fig. 4).

Effects of Using Different Definitions of Inpatient and Outpatient Status on Postoperative Outcomes for THA

After controlling for potential confounding factors such as gender, body mass index, functional status before surgery, comorbidities, and smoking status, the following variables were found to be greater for "inpatient" THA compared with "outpatient" THA: any adverse event (relative risk, $2.643[98.75 \%$ confidence interval $\{\mathrm{CI}\}, 1.225-5.702] ; \mathrm{p}=$

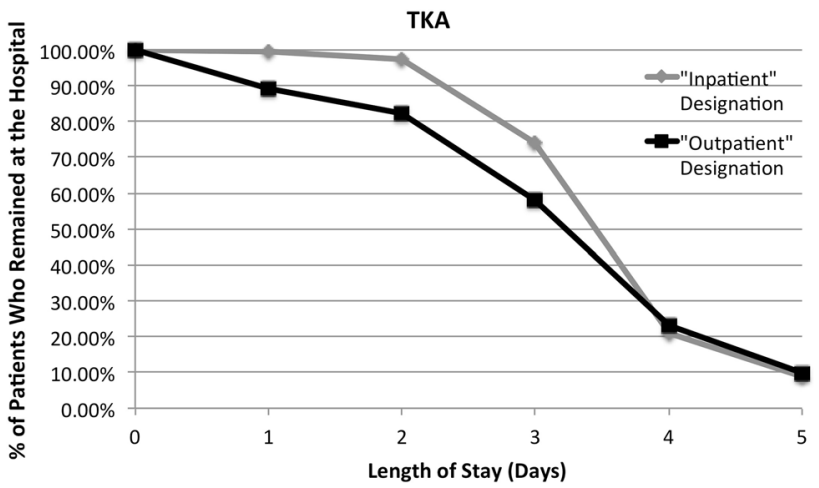

Fig. 4 Kaplan-Meier curve shows the percentage of patients who remained at the hospital at a particular LOS after receiving "inpatient" or "outpatient" TKA. Y-axis represents the percentage of patients who remained at the hospital. X-axis represents the LOS.

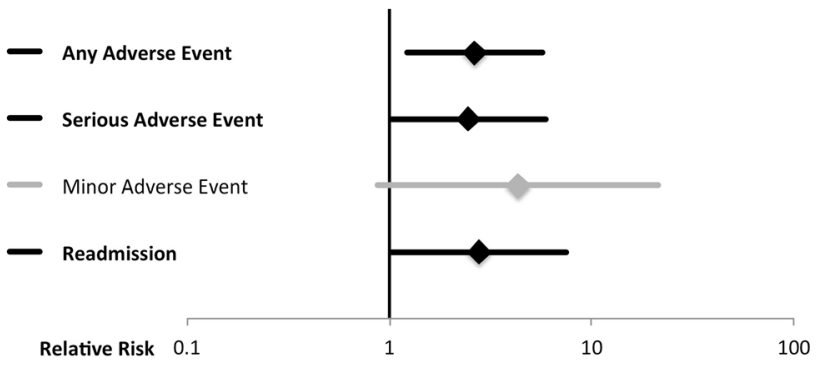

Fig. 5 Relative risk of adverse events is shown using the ACSNSQIP "inpatient"/"outpatient" classification for THA: "inpatient" relative to "outpatient." Adverse events listed at the left. Diamonds central to the horizontal lines indicate relative risks. Horizontal lines denote the $98.75 \%$ CIs of those relative risks per Bonferroni's correction. The vertical line indicates a relative risk of 1 . Therefore, horizontal lines that cross the vertical line indicate relative risks that are not statistically significant. Black lines and bolding indicate relative risks that are statistically significant.

0.002), SAE (relative risk, 2.455 [98.75\% CI, 1.0185.921]; $\mathrm{p}=0.011$ ), and readmission (relative risk, 2.775 [98.75\% CI, 1.025-7.509]; p = 0.010) (Fig. 5). The frequency of postoperative adverse events and readmission for patients who underwent THA is reported (Appendix 1).

For the same procedure and controlling for the same factors, patients who had LOS $>0$ were not associated with any increased risk for any adverse event, SAE, MAE, or readmission compared with patients who had $\mathrm{LOS}=0$ (Fig. 6).

Effects of Using Different Definitions of Inpatient and Outpatient Status on Postoperative Outcomes for TKA

After controlling for potential confounding factors such as gender, body mass index, functional status before surgery, 


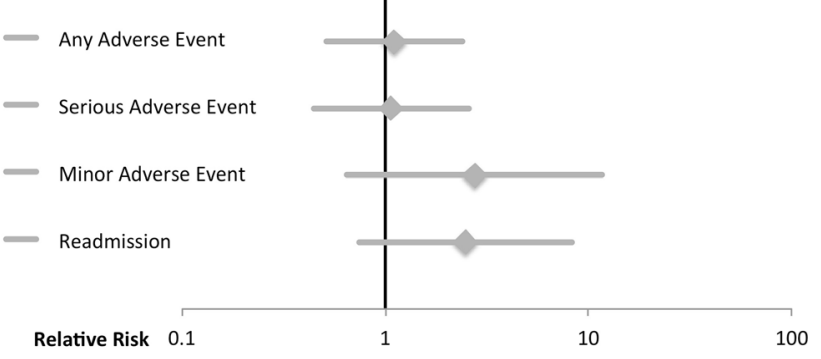

Fig. 6 Relative risk of adverse events is shown using the length of stay variable definition for THA: LOS $>0$ (inpatient) relative to LOS $=0$ (outpatient). Adverse events listed at the left. Diamonds central to the horizontal lines indicate relative risks. Horizontal lines denote the 98.75\% CIs of those relative risks per Bonferroni's correction. The vertical line indicates a relative risk of 1 . Therefore, horizontal lines that cross the vertical line indicate relative risks that are not statistically significant. Black lines and bolding indicate relative risks that are statistically significant.

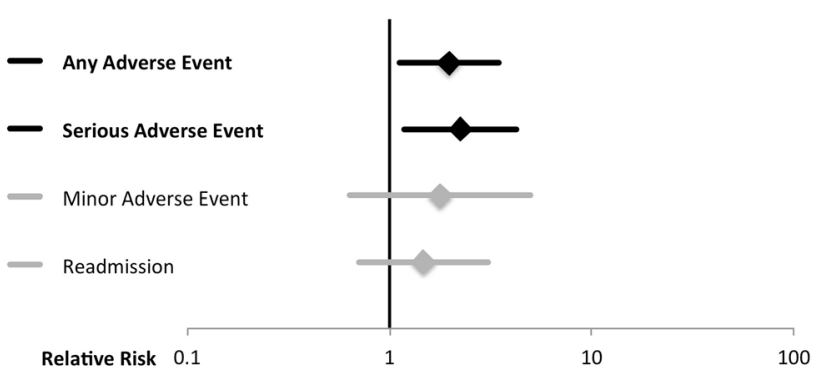

Fig. 7 Relative risk of adverse events is shown using the ACSNSQIP "inpatient"/" outpatient" classification for TKA: "inpatient" relative to "outpatient." Adverse events are listed at the left. Diamonds central to the horizontal lines indicate relative risks. Horizontal lines denote the $98.75 \%$ CIs of those relative risks per Bonferroni's correction. The vertical line indicates a relative risk of 1 . Therefore, horizontal lines that cross the vertical line indicate relative risks that are not statistically significant. Black lines and bolding indicate relative risks that are statistically significant.

comorbidities, and smoking status, the following variables were found to be greater for "inpatient" TKA compared with "outpatient" TKA: any adverse event (relative risk, 1.964 [98.75\% CI, 1.113-3.466]; $\mathrm{p}=0.003)$ and SAE (relative risk, 2.238 [98.75\% CI, 1.174-4.266]; $\mathrm{p}=0.002$ ) (Fig. 7). The frequency of postoperative adverse events and readmission for patients who underwent TKA is reported (Appendix 1).

For the same procedure and controlling for the same factors, patients who had LOS $>0$ were not associated with any increased risk for any adverse event, SAE, MAE, or readmission compared with patients who had LOS $=0$ (Fig. 8).

\section{Discussion}

Multiple studies in orthopaedics have used NSQIP, a large national database that provides an "outpatient" status

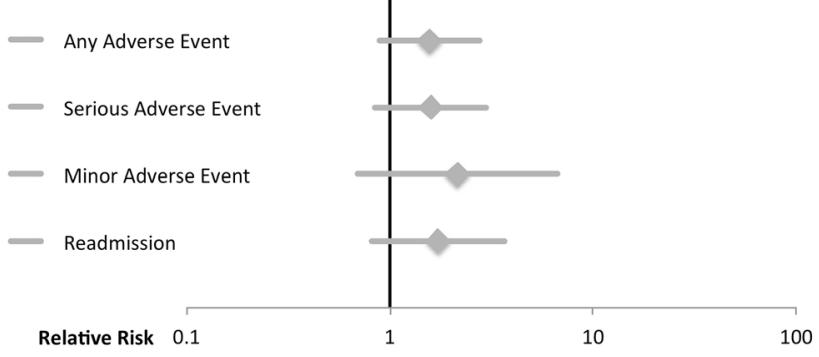

Fig. 8 Relative risk of adverse events is shown using the length of stay variable definition for TKA: $\operatorname{LOS}>0$ (inpatient) relative to LOS $=$ 0 (outpatient). Adverse events are listed at the left. Diamonds central to the horizontal lines indicate relative risks. Horizontal lines denote the $98.75 \%$ CIs of those relative risks per Bonferroni's correction. The vertical line indicates a relative risk of 1 . Therefore, horizontal lines that cross the vertical line indicate relative risks that are not statistically significant. Black lines and bolding indicate relative risks that are statistically significant.

variable, to study outpatient procedures without determining what, exactly, "outpatient" means in NSQIP $[32,35,41]$. Under current regulations in the United States, patients who underwent outpatient surgery may stay one or more nights at the hospital under "observation" status despite being coded as an outpatient [38]. It is important to determine the degree to which this is the case in NSQIP because this may influence the way clinicians interpret results from that source and the way hospital systems and policymakers use those data. The primary aim of the present study was to utilize NSQIP to characterize the definition of "inpatient" and "outpatient" status for THAs and TKAs in comparison to actual hospital LOS. The secondary aim was to assess if using different definitions of hospital stay, stated status versus actual LOS, would lead to different results when examining risk for postoperative adverse events after THA and TKA. The relevance of these research aims is important because not only is the number of these procedures expected to rise with an aging US population, but also because the number of studies investigating outpatient arthroplasty is expected to rise as a result of efforts to improve levels of patient satisfaction and reduce cost $[15,26,29]$.

Limitations of the present study are inherent to data sets and include concern regarding data accuracy, followup only through the 30th postoperative day, potential for underreporting of postdischarge adverse events, and lack of procedure-specific outcomes. That being said, NSQIP undergoes interrater reliability audits to ensure that it contains high-quality data [1]. Furthermore, the grouping of specific adverse events into broad categories, which may misrepresent the severity of some complications, and not distinguishing among adverse events that occurred before hospital discharge and those that occurred after discharge, 
are limitations in this study. Another limitation is the limited resolution of the LOS $=0$ parameter in this study. Because NSQIP only provides LOS in days, this study is unable to specify the number of hours from admission to discharge. An additional limitation is the lack of rigorous statistical power analysis in this study. Because this study is a retrospective study, a priori power analysis, which is universally accepted, is not possible. Retrospective power analysis was not performed because it remains controversial and can lead to biased power estimates with low precision [14, 17]. Instead, confidence intervals are provided in this study [17]. Strengths of this analysis include the clinical relevance of the research aims. The recent changes in healthcare insurance and the aging US population make the appropriate exploration of outpatient procedures in terms of safety and cost-effectiveness more important than ever. The large number of recent orthopaedic studies comparing inpatient and outpatient procedures underscores this importance $[2,5,9,10,15$, 16, 24, 25, 29, 30, 37, 40, 48].

Contrary to the intuitive definition, this study demonstrated that the definition of "inpatient" and "outpatient" status used by hospitals throughout the United States often is not synonymous with $\operatorname{LOS}>0$ and $\operatorname{LOS}=0$. This was true for both patients undergoing THA and those undergoing TKA in which $22 \%$ and $23 \%$ of patients, respectively, who underwent "outpatient" procedures still remained at the hospital 4 days after admission. Certainly a portion of the cost-saving benefits of true outpatient procedures stems from the patient not staying any nights in the hospital. Furthermore, to accurately evaluate the safety and efficacy of traditionally defined outpatient arthroplasty, a quantitative and consistent definition, in terms of actual hospital LOS, would seem to be important.

One potential reason that the "inpatient"/"outpatient" status does not correlate with $\operatorname{LOS}>0 / \mathrm{LOS}=0$ is understood through the idea of observation status. Observation status is technically an outpatient visit, although the patient can spend multiple nights in the hospital. The use of this designation may stem from Medicare auditors denying the more heavily reimbursed short-term hospital admission but providing less scrutiny to the less compensated observation status, thus incentivizing hospitals to pursue this option in cases in which they believe admission reimbursement is not possible [51]. In fact, in recent years, the Centers for Medicare \& Medicaid Services have observed a higher frequency of beneficiaries being treated as hospital outpatients and receiving extended "observation" services [13].

Despite the definitional differences, certain past orthopaedic data set studies relied on the nebulous "inpatient"/ "outpatient" NSQIP variable in their analysis as opposed to LOS $>0 / \operatorname{LOS}=0[32,35,41,50]$. When a comparison was done using admission status as a predictor of adverse events after THA, an "inpatient" assignment in comparison to an "outpatient" assignment was found to be associated with an increased risk of any adverse event, SAE, and readmission (Fig. 5). When the same analysis was repeated comparing LOS $>0$ with $\operatorname{LOS}=0$, there was no observed difference in relative risk (Fig. 6). The same trend existed for a TKA analysis in which "inpatient" assignment versus "outpatient" status was associated with an increased risk of any adverse event and SAE (Fig. 7). This association was not present when comparing $\operatorname{LOS}>0$ with $\operatorname{LOS}=0$ (Fig. 8). The discrepancies in these findings may be because the "inpatient"/“outpatient" assignment was assigned before surgery. Patients who had more comorbid conditions that this study was unable to control for could be assigned to the "inpatient" status because they may require more resources, thus predisposing "inpatient" surgery as a risk factor for adverse events. Another possible explanation may be the result of the groupings of adverse events used in this study. Additional possible explanations for these discrepancies could be attributable to slight differences in the statistical power between the "inpatient"/"outpatient" and "LOS >0"/"LOS $=0$ " cohorts and other clinical factors that this study did not include and could not control for.

In summary, the hospital-assigned "inpatient"/“outpatient" status is not equivalent to $\operatorname{LOS}>0 / \mathrm{LOS}=0$ and the use of different definitions of inpatient versus outpatient may lead to different assignments of risk factors for postoperative adverse outcomes. Accurate data on risk factors for complications after total joint arthroplasty are crucial for efforts to reduce length of hospital stay and minimize complications after surgery. This study highlights the importance of consistently quantifying outpatient procedures based on actual hospital LOS for future investigations, calls for careful interpretation of existing studies on outpatient procedures that did not provide a quantitative definition, and underscores the need to closely scrutinize variables in large data sets before utilizing them.

\section{References}

1. ACS-NSQIP. User Guide for the 2013 ACS NSQIP Participant Use Data File. 2014. Available at: http://site.acsnsqip.org/wpcontent/uploads/2014/11/ACS_NSQIP_PUF_User_Guide_2013.pdf. Accessed June 5, 2016.

2. Aynardi M, Post Z, Ong A, Orozco F, Sukin DC. Outpatient surgery as a means of cost reduction in total hip arthroplasty: a case-control study. HSS J. 2014;10:252-255.

3. Berger RA, Cross MB, Sanders S. Outpatient hip and knee replacement: the experience from the first 15 years. Instr Course Lect. 2016;65:547-551.

4. Berger RA, Kusuma SK, Sanders SA, Thill ES, Sporer SM. The feasibility and perioperative complications of outpatient knee arthroplasty. Clin Orthop Relat Res. 2009;467:1443-1449. 
5. Bertin KC. Minimally invasive outpatient total hip arthroplasty: a financial analysis. Clin Orthop Relat Res. 2005;435:154-163.

6. Bohl DD, Fu MC, Golinvaux NS, Basques BA, Gruskay JA, Grauer JN. The 'July effect' in primary total hip and knee arthroplasty: analysis of 21,434 cases from the ACS-NSQIP database. J Arthroplasty. 2014;29:1332-1338.

7. Bohl DD, Russo GS, Basques BA, Golinvaux NS, Fu MC, Long WD 3rd, Grauer JN. Variations in data collection methods between national databases affect study results: a comparison of the nationwide inpatient sample and national surgical quality improvement program databases for lumbar spine fusion procedures. J Bone Joint Surg Am. 2014;96:e193.

8. Charlson ME, Pompei P, Ales KL, MacKenzie CR. A new method of classifying prognostic comorbidity in longitudinal studies: development and validation. J Chronic Dis. 1987;40:373-383.

9. Cross MB, Berger R. Feasibility and safety of performing outpatient unicompartmental knee arthroplasty. Int Orthop. 2014;38:443-447.

10. Dorr LD, Thomas DJ, Zhu J, Dastane M, Chao L, Long WT. Outpatient total hip arthroplasty. J Arthroplasty. 2010;25:501506.

11. Dunn OJ. Multiple comparisons among means. J Am Stat Assoc. 1961;56:52-64.

12. Ehlert BA, Nelson JT, Goettler CE, Parker FM, Bogey WM, Powell CS, Stoner MC. Examining the myth of the 'July phenomenon' in surgical patients. Surgery. 2011;150:332-338.

13. Fact Sheet: Two-Midnight Rule. Available at: https://www.cms. gov/Newsroom/MediaReleaseDatabase/Fact-sheets/2015-Fact-sheetsitems/2015-07-01-2.html. Accessed October 3, 2016.

14. Gerard PD, Smith DR, Weerakkody G. Limits of retrospective power analysis. Journal of Wildlife Management. 1998;62:801807.

15. Goyal N, Chen AF, Padgett SE, Tan TL, Kheir MM, Hopper RH Jr, Hamilton WG, Hozack WJ. Otto Aufranc Award: A multicenter, randomized study of outpatient versus inpatient total hip arthroplasty. Clin Orthop Relat Res. 2016 Jun 10. [Epub ahead of print].

16. Hartog YM, Mathijssen NM, Vehmeijer SB. Total hip arthroplasty in an outpatient setting in 27 selected patients. Acta Orthop. 2015;86:667-670.

17. Hoenig JM, Heisey DM. The abuse of power. Am Stat. 2001;55:19-24.

18. Inacio MC, Chen Y, Paxton EW, Namba RS, Kurtz SM, Cafri G. Statistics in Brief: An introduction to the use of propensity scores. Clin Orthop Relat Res. 2015;473:2722-2726.

19. Jain U, Chandra RK, Smith SS, Pilecki M, Kim JY. Predictors of readmission after outpatient otolaryngologic surgery. Laryngoscope. 2014;124:1783-1788.

20. Khavanin N, Mlodinow A, Kim JY, Ver Halen JP, Antony AK, Samant S. Assessing safety and outcomes in outpatient versus inpatient thyroidectomy using the NSQIP: a propensity score matched analysis of 16,370 patients. Ann Surg Oncol. 2015;22:429-436.

21. Khavanin N, Mlodinow A, Kim JY, Ver Halen JP, Samant S. Predictors of 30-day readmission after outpatient thyroidectomy: an analysis of the 2011 NSQIP data set. Am J Otolaryngol. 2014:35:332-339.

22. Khavanin N, Mlodinow A, Milad MP, Bilimoria KY, Kim JY. Comparison of perioperative outcomes in outpatient and inpatient laparoscopic hysterectomy. J Minim Invasive Gynecol. 2013;20:604-610.

23. Khuri SF. The NSQIP: a new frontier in surgery. Surgery. 2005; $138: 837-843$.

24. Kolisek FR, McGrath MS, Jessup NM, Monesmith EA, Mont MA. Comparison of outpatient versus inpatient total knee arthroplasty. Clin Orthop Relat Res. 2009;467:1438-1442.
25. Kort NP, Bemelmans YF, Schotanus MG. Outpatient surgery for unicompartmental knee arthroplasty is effective and safe. Knee Surg Sports Traumatol Arthrosc. 2015 Jul 1. [Epub ahead of print].

26. Kurtz S, Ong K, Lau E, Mowat F, Halpern M. Projections of primary and revision hip and knee arthroplasty in the United States from 2005 to 2030. J Bone Joint Surg Am. 2007;89:780785 .

27. Lim S, Jordan SW, Jain U, Kim JY. Predictors and causes of unplanned re-operations in outpatient plastic surgery: a multiinstitutional analysis of 6749 patients using the 2011 NSQIP database. J Plast Surg Hand Surg. 2014;48:270-275.

28. Lipira AB, Sood RF, Tatman PD, Davis JI, Morrison SD, Ko JH. Complications within 30 days of hand surgery: an analysis of 10,646 patients. J Hand Surg Am. 2015;40:1852-1859.e1853.

29. Lovald ST, Ong KL, Malkani AL, Lau EC, Schmier JK, Kurtz SM, Manley MT. Complications, mortality, and costs for outpatient and short-stay total knee arthroplasty patients in comparison to standard-stay patients. $J$ Arthroplasty. 2014;29:510-515.

30. Lovecchio F, Alvi H, Sahota S, Beal M, Manning D. Is outpatient arthroplasty as safe as fast-track inpatient arthroplasty? A propensity score matched analysis. $J$ Arthroplasty. 2016;31(Suppl):197-201.

31. Lukasiewicz AM, Basques BA, Bohl DD, Webb ML, Samuel AM, Grauer JN. Myelopathy is associated with increased all-cause morbidity and mortality following anterior cervical discectomy and fusion: a study of 5256 patients in American College of Surgeons National Surgical Quality Improvement Program (ACS-NSQIP). Spine (Phila Pa 1976). 2015;40:443-449.

32. Martin CT, Pugely AJ, Gao Y, Mendoza-Lattes S. Thirty-day morbidity after single-level anterior cervical discectomy and fusion: identification of risk factors and emphasis on the safety of outpatient procedures. J Bone Joint Surg Am. 2014;96:12881294.

33. Mason RJ, Moazzez A, Sohn HJ, Berne TV, Katkhouda N. Laparoscopic versus open anterior abdominal wall hernia repair: 30-day morbidity and mortality using the ACS-NSQIP database. Ann Surg. 2011;254:641-652.

34. Massenburg BB, Sanati-Mehrizy P, Jablonka EM, Taub PJ. The impact of resident participation in outpatient plastic surgical procedures. Aesthetic Plast Surg. 2016;40:584-591.

35. McGirt MJ, Godil SS, Asher AL, Parker SL, Devin CJ. Quality analysis of anterior cervical discectomy and fusion in the outpatient versus inpatient setting: analysis of 7288 patients from the NSQIP database. Neurosurg Focus. 2015;39:E9.

36. Mioton LM, Alghoul MS, Kim JY. A comparative analysis of readmission rates after outpatient cosmetic surgery. Aesthet Surg J. 2014;34:317-323.

37. Otero JE, Gholson JJ, Pugely AJ, Gao Y, Bedard NA, Callaghan JJ. Length of hospitalization after joint arthroplasty: does early discharge affect complications and readmission rates? J Arthroplasty. 2016;31:2714-2725.

38. Outpatient Observation Services. Available at: https://www.cms. gov/Regulations-and-Guidance/Guidance/Transmittals/downloads/ r42bp.pdf. Accessed August 25, 2016.

39. Pannucci CJ, Shanks A, Moote MJ, Bahl V, Cederna PS, Naughton NN, Wakefield TW, Henke PK, Campbell DA, Kheterpal S. Identifying patients at high risk for venous thromboembolism requiring treatment after outpatient surgery. Ann Surg. 2012;255:1093-1099.

40. Parcells BW, Giacobbe D, Macknet D, Smith A, Schottenfeld M, Harwood DA, Kayiaros S. Total joint arthroplasty in a standalone ambulatory surgical center: short-term outcomes. Orthopedics. 2016;39:223-228.

41. Pugely AJ, Martin CT, Gao Y, Mendoza-Lattes SA. Outpatient surgery reduces short-term complications in lumbar discectomy: 
an analysis of 4310 patients from the ACS-NSQIP database. Spine (Phila Pa 1976). 2013;38:264-271.

42. Qin C, Antony AK, Aggarwal A, Jordan S, Gutowski KA, Kim JY. Assessing outcomes and safety of inpatient versus outpatient tissue expander immediate breast reconstruction. Ann Surg Oncol. 2015;22:3724-3729.

43. Qin C, Hackett NJ, Kim JY. Assessing the safety of outpatient ventral hernia repair: a NSQIP analysis of 7666 patients. Hernia. 2015;19:919-926.

44. Rao A, Polanco A, Qiu S, Kim J, Chin EH, Divino CM, Nguyen SQ. Safety of outpatient laparoscopic cholecystectomy in the elderly: analysis of 15,248 patients using the NSQIP database. $J$ Am Coll Surg. 2013;217:1038-1043.

45. Raval MV, Hamilton BH, Ingraham AM, Ko CY, Hall BL. The importance of assessing both inpatient and outpatient surgical quality. Ann Surg. 2011;253:611-618.

46. Sacco Casamassima MG, Gause C, Yang J, Goldstein SD, Swarup A, Abdullah F. Safety of outpatient laparoscopic cholecystectomy in children: analysis of 2050 elective ACS NSQIPpediatric cases. Pediatr Surg Int. 2016;32:541-551.
47. Schilling PL, Hallstrom BR, Birkmeyer JD, Carpenter JE. Prioritizing perioperative quality improvement in orthopaedic surgery. J Bone Joint Surg Am. 2010;92:1884-1889.

48. Schotanus MG, Bemelmans YF, Grimm B, Heyligers IC, Kort NP. Physical activity after outpatient surgery and enhanced recovery for total knee arthroplasty. Knee Surg Sports Traumatol Arthrosc. 2016 Aug 4. [Epub ahead of print].

49. Sundararajan V, Henderson T, Perry C, Muggivan A, Quan H, Ghali WA. New ICD-10 version of the Charlson comorbidity index predicted in-hospital mortality. $J$ Clin Epidemiol. 2004;57:1288-1294.

50. Thibaudeau S, Anari JB, Carducci N, Carrigan RB. 30-day readmission after pediatric upper extremity surgery: Analysis of the NSQIP database. J Pediatr Surg. 2016;51:1370-1374.

51. Weaver C. MAW. Medicare rules reshape hospital admissions. The Wall Street Journal. December 1, 2015.

52. Zou G. A modified poisson regression approach to prospective studies with binary data. Am J Epidemiol. 2004;159:702-706. 